\title{
Treatment of chronic immune thrombocytopenic purpura in adults
}

\author{
Bertrand Godeau • Marc Michel
}

Received: 1 August 2009 /Accepted: 22 March 2010/Published online: 14 April 2010

(C) Springer-Verlag 2010

\begin{abstract}
Until recently, the management of refractory immune thrombocytopenic purpura (ITP) was a real challenge as shown by the large variety of treatments proposed in both American Society of Hematology and the British Committee for Standards in Haematology guidelines published 10 and 6 years ago, respectively. However, as in the past 5 years, new therapeutic approaches including eradication of Helicobacter pylori in infected patients and rituximab have been proposed and the thrombopoietinreceptor agonists have been developed and licensed for ITP. It is likely that the therapeutic strategy of ITP will be profoundly modified and revisited in the next future. The purpose of this article is to discuss the impact and the place of these new therapeutic options into the whole treatment strategy of chronic ITP and to draw the perspective of new experimental therapies.
\end{abstract}

Keywords Immune thrombocytopenic purpura .

Rituximab · Thrombopoietin-receptor agonist .

Helicobacter pylori $\cdot$ Romiplostim $\cdot$ Eltrombopag

\section{Introduction}

Primary immune thrombocytopenia (ITP), previously referred to as idiopathic thrombocytopenic purpura, is an immune-mediated acquired disorder characterized by isolated thrombocytopenia, defined as a peripheral blood

B. Godeau $(\bowtie) \cdot$ M. Michel

Centre de référence labélisé de prise en charge des cytopénies autoimmunes de l'adulte, service de médecine interne, CHU Henri Mondor, Assistance-Publique Hôpitaux de Paris, Université Paris 12,

94000 Créteil, France

e-mail: bertrand.godeau@hmn.aphp.fr platelet count $<100 \times 10^{9} / \mathrm{L}$ in the absence of any other cause of the thrombocytopenia [1]. ITP is an orphan disease that can occur at any age with an overall incidence of 1.6 to $3.9 / 100,000$ persons $[2,3]$. In children, it is usually a selflimited disease which spontaneously recovers within a few weeks. In contrast, about $70 \%$ of adults remain thrombocytopenic after 6 to 12 months and thus develop chronic ITP. The signs and symptoms of ITP vary widely. Many patients have either no symptoms or minimal bruising, while others experience serious bleeding. The overall prognosis of ITP is good with a mortality rate less than $2 \%$ [4], but mortality can rise up to $10 \%$ in a subgroup of selected patients refractory to splenectomy [5].

ITP treatment has long been based mainly on empirical data with a few controlled studies [6]. Due to these limitations, it has been difficult to establish robust guidelines for the treatment of ITP. Nevertheless, ITP guidelines were produced by the American Society of Hematology (ASH) more than 10 years ago [7] and the British Committee for Standards in Haematology (BCSH) 6 years ago [8]. ASH and BCSH guidelines emphasize that the aim of treatment is primarily to prevent bleeding and avoid the side effects of therapy and not to normalize the platelet count. Usually, only patients with a platelet count of $<30 \times$ $10^{9} / \mathrm{L}$ and bleeding symptoms require treatment. Guidelines suggest corticosteroids as the first-line treatment. The use of IVIg or IV anti-D may be appropriate mainly in patients who present with severe bleeding symptoms. The effects of these first-line treatments are however often only transient, and splenectomy is proposed in both guidelines as the major second-line therapy. Patients who do not respond to splenectomy are defined as having refractory ITP.

Until recently, the management of refractory ITP was a real challenge as shown by the large variety of treatments proposed in both ASH and BCSH guidelines. However, as 
in the past 5 years, new therapeutic approaches (eradication of Helicobacter pylori, rituximab...) have been proposed and the thrombopoietin-receptor (TPO-r) agonists have been developed and licensed for ITP. It is likely that the therapeutic strategy of ITP will be profoundly modified and revisited in the near future. The purpose of this article is to discuss the impact and the place of these new therapeutic options into the whole treatment strategy of chronic ITP and to draw the perspective of new experimental therapies.

\section{Helicobacter pylori and ITP}

Helicobacter pylori is a Gram-negative bacterium that colonizes the human stomach with a great variation of prevalence across the world. For example, the prevalence in Japan and Italy is greater than $50 \%$ while it is slightly less than $30 \%$ in France and even lower in the USA (about $20 \%)$. H. pylori infection has been suspected to be associated with a large spectrum of extra-intestinal diseases including various autoimmune disorders.

The relationship between $H$. pylori infection and ITP was first described 10 years ago by Gasbarrini et al. [9]. The rate of $H$. pylori infection in patients with ITP varies greatly from country to country, but this prevalence was not found different from that reported in the general healthy population matched for age and geographical area [10]. The clinical characteristics of $H$. pylori-associated ITP appear similar to those observed in non-infected ITP patients and are rarely severe.

The mechanism of $H$. pylori-induced thrombocytopenia is far from being established and many hypotheses have been raised [10]. One of them is molecular mimicry; $H$. pylori induce the production of a variety of antibodies, some of which could cross-react towards various platelet glycoprotein antigens. A protein named $\mathrm{CagA}$, which is a virulence factor of $H$. pylori, can induce the production of antibodies that cross-react with a peptide specifically expressed by platelets of patients with ITP [11]. The proportion of CagA-positive strains of $H$. pylori varies depending upon geographic location [10]. This finding could explain why the association of ITP and H. pylori is stronger in some countries such as Japan and Italy.

Whether the eradication of $H$. pylori infection can increase the platelet count in patients with ITP is still a controversial issue [10]. Stasi et al. [12] conducted a systematic review of the literature including 1,555 patients. With a combination of amoxicillin, clarithromycin, and a proton pump inhibitor usually given for 1 to 2 weeks, an overall response defined by a platelet count of $\geq 30 \times 10^{9} / \mathrm{L}$ and at least doubling of the platelet count at baseline was observed in $50 \%$ of the cases. Reducing the analysis to the patients with a baseline platelet count $\leq 30 \times 10^{9} / \mathrm{L}$, the overall response rate was only $35 \%$. The response occurred most commonly 2 weeks after the completion of eradication therapy whereas the long term response is not well known. The response rates were much higher in countries where the rate of $H$. pylori carriage is high. No severe side effects of the treatment occurred.

In view of these conflicting results, it is difficult to draw a consensual proposal concerning the interest of a systematic $H$. pylori screening in ITP patients. The screening would imply the use of the breath test or stool antigen tests as opposed to antibody testing in order to confirm H. pylori carriage. The non-invasiveness of diagnostic methods and the good cost/effectiveness ratio and safety profile of the standard eradication regimen should argue for a systematic screening of adults diagnosed with ITP and for an eradication treatment in $H$. pylori-positive patients. Ideally, it must be confirmed by a prospective randomized study that should include a large cohort of patients with various ethnical origins to be sure that the results could be adapted in all countries.

\section{Rituximab}

Rituximab is a chimeric monoclonal anti-CD20 antibody that is currently indicated for the treatment of lymphoma. Because of its ability to deplete autoantibody-producing B lymphocytes and its apparently good safety profile, it has been used in patients with various autoimmune diseases such as lupus and rheumatoid arthritis [13]. Its efficacy in ITP was first reported almost 10 years ago [14] and it is now commonly used off-label in many countries in this setting, especially in patients who are refractory to splenectomy or in whom splenectomy is contra-indicated. A systematic review of the literature showed that rituximab resulted in complete response (platelet count of $>150 \times 10^{9} / \mathrm{L}$ ) in $44 \%$ of patients and an overall response (platelet count of $>50 \times 10^{9} / \mathrm{L}$ ) in $62 \%$ [15]. Responses generally occur after $1-$ 2 weeks to $6-8$ weeks.

An open non-randomized prospective study conducted in non-splectomized patients with chronic ITP showed that, after 2 years of observation, $40 \%$ of patients had a platelet count of $>30 \times 10^{9} / \mathrm{L}$ without treatment, suggesting that rituximab is an apparently safe splenectomy-avoiding option in some adults with chronic ITP [16]. A prospective randomized Italian study comparing dexamethasone alone with dexamethasone plus rituximab in adults with newly diagnosed ITP confirmed the interest of rituximab in nonsplectomized patients by showing a better response rate in the group of patients receiving rituximab with more than $60 \%$ of response after 2 years of follow-up [17].

However, despite these promising results, several points remain unresolved. Should the lymphoma regimen (i.e., 
$375 \mathrm{mg} / \mathrm{m}^{2} /$ week $\times 4$ ) be the one used for ITP ? What are the mechanisms of action of rituximab? Are there predictive factors of response? What is the long-term response and can we really cure ITP with rituximab? Is the treatment really safe?

Recent data provide some answers to these important questions.

Two open non-controlled studies used a fixed low dose of $100 \mathrm{mg}$ weekly for four times in ITP [18, 19]. A complete and durable response was observed in about $60 \%$ of patients and efficacy of the treatment appeared comparable to that observed with the standard dose of the drug. Recently, a promising response was also reported with a low dose of veltuzumab, a new humanized anti-CD20 monoclonal antibody. Administered via only two intravenous or subcutaneous administrations, a sustained response was observed in $60 \%$ patients with chronic ITP [20]. However, the non-randomized design of these studies and the low number of patients included prevent one from drawing definite conclusion concerning the optimal dose of rituximab.

The mechanisms of action of rituximab are far from being completely known. However, Stasi et al. [21] demonstrated that there were several abnormalities of $\mathrm{T}$ cell subsets in ITP patients including an increase of the $\mathrm{Th} 1 / \mathrm{Th} 2$ ratio and an expansion of oligoclonal $\mathrm{T}$ cells that could be reverted by a $\mathrm{B}$ cell depletion induced by rituximab. Stasi et al. [22] also showed that ITP patients had a reduced number and a defective suppressive capacity of $\mathrm{T}$ regs that could be restored after rituximab.

No clear predictive factors of responses of rituximab were determined, but younger age was associated with a better long-term good response [16] and early administration might have a better disease-modifying effect [21, 23]. It has been demonstrated that chronic ITP is associated with the expansion of oligoclonal $\mathrm{T}$ cells driving autoantibody production not suppressed by Tregs and with the expansion of self-reactive clones resistant to Fas-mediated cell death $[21,24,25]$. It is possible that oligoclonal $\mathrm{T}$ cells are less sensitive to rituximab that could argue for an early administration of this treatment in ITP.

The long-term efficacy of rituximab remains undetermined, but it has been shown that in a subset of long-term responders to rituximab about $20 \%$ of patients remained in remission after a follow-up of 5 years, suggesting that rituximab may definitely have a curative effect in ITP $[26,27]$.

Rituximab appears to have a good safety profile $[16,17$, 28]. However, we cannot exclude that the long-lasting impairment of humoral immunity could lead to an increase risk of viral and/or bacterial infections. Hypogammaglobulinia has been observed, particularly after multiple doses, in patients treated for rheumatic disorders [29]. Even if this risk is not well known in patients treated with rituximab for
ITP, multiple doses should be avoided in the case of relapse after a first cure and it is best to test the baseline immunoglobulins level prior to administering the treatment. Rituximab can induce hepatitis B virus (HBV) reactivation and fatal hepatitis has been reported. Rituximab is then contra-indicated in HBV-infected patients [30]. Several cases of fatal progressive multifocal leukoencephalopathy have been reported in patients with auto-immune diseases treated with rituximab, including one patient with ITP [31, 32]. An international registry could be useful to better assess the incidence of this very rare but life-threatening opportunistic infection.

\section{TPO-receptor agonists}

ITP has long been considered to be only a matter of accelerated platelet destruction. However, a number of lines of evidence have come, showing that ITP is also a matter of impaired platelet production [33]. Suboptimal platelet production would be consistent with an important role for thrombopoietic agents in the management of ITP.

To date, two TPO-r agonists have been developed and licensed in ITP.

Romiplostim (AMG531, AMGEN) is a peptidobody consisting of two covalently linked carrier-Fc domains, each attached to a peptide containing many c-MPLactivating sequences. It is administered subcutaneously at weekly intervals. In pivotal studies conducted in splenectomized and non-splenectomized patients, overall response was observed in more than $80 \%$ of patients with chronic ITP [34]. Durable responses were seen in $61 \%$ of nonsplenectomized patients and $38 \%$ of splenectomized patients. Platelet response was maintained by most patients during long-term treatment with romiplostim for up to 3 or 4 years in an open-label extension study [35].

In the USA, the FDA approved romiplostim as a longterm treatment for chronic ITP in adults who have not responded to standard treatments, such as corticosteroids, intravenous immunoglobulin, anti-Rho(D) immune globulin, or splenectomy. In Europe, the drug has obtained a license only in adult patients with chronic ITP who have failed to respond after splenectomy. Romiplostim may be considered as a second-line treatment for adult nonsplenectomized ITP patients if surgery is contra-indicated.

Eltrombopag (promacta, GSK) is an oral, non-peptidic small molecule and a thrombopoietin-receptor agonist that is given orally once daily. In a randomized placebocontrolled trial compared with the effect with placebo, more than $70 \%$ of patients responded to the treatment over a 6-week period [36]. In an extended phase III study, almost $80 \%$ of patients maintained a platelet count of greater than $50 \times 10^{9} / \mathrm{L}$ for more than half their time in the study [37] 
Romiplostim and eltrombopag appear to be well tolerated and no severe side effects were observed in the published studies including hundreds of patients. However, several potential adverse consequences of thrombopoietic growth factors are a matter of concern [33]. Thrombocytosis may occasionally occur with these drugs that could be associated with an increased risk of thrombosis. Reticulin deposition has been observed with both eltrombopag and romiplostim. It appears dose dependent [35]. In the absence of systematic bone marrow examination, its real incidence remains however unknown and could be underestimated. This side effect could be a problem in case of long-term use, particularly in young patients.

In summary, these agents appear unequivocally very active in ITP. How they impact the management of ITP remains to be seen, but they have some of the characteristics required for widespread usage: high rate of efficacy, low degree of toxicity. However, these treatments are costly and should be only considered as "supportive" therapy as due to their mechanism of action the platelet count usually decreases back to the pre-treatment level within 2 weeks following treatment withdrawal.

\section{Experimental therapies}

With the better understanding of the pathophysiology of ITP, new therapeutic strategies are being developed. Among them, syk tyrosine-kinase inhibitors and humanized antiCD40L monoclonal antibodies could be useful [38-40].

\section{Conclusion}

Ten years ago, the ASH published ITP practice guidelines; we now benefit from new drugs tested in prospective clinical trials including multicenter, randomized studies. It is likely that the therapeutic strategy of ITP will be profoundly modified in the near future. It remains however difficult to propose a consensual and uniform therapeutic strategy at this stage as some of new treatments have only been recently licensed and pharmako-economics considerations could also influence our daily practice. Corticosteroids remain the first-line treatment and IVIg and anti-D are still indicated in case of severe bleeding manifestations since new drugs such as rituximab and TPO-r agonists do not increase the platelet count as quickly as steroids and immunoglobulin preparations do. Splenectomy has long been the "gold standard" second-line treatment for patients with persisting or chronic severe ITP, but it has now become obvious that an increasing number of both clinicians and patients are reluctant to consider/undergo splenectomy. Rituximab has become a possible alternative to splenectomy, although the durability of response and its long-term safety is still a matter of debate and its use in an off-label setting has become a real issue in many countries now that new drugs have been licensed for ITP [41]. The efficacy of TPO-r agonists is unquestionable as clearly shown by several well-designed controlled randomized studies. These treatments however do not modify the natural course of the disease as they are not supposed to reverse the autoimmune process. They should therefore not be compared and balanced with other treatment strategies such as splenectomy and rituximab which aim to induce long-term remission or even to cure the disease [33]. Therefore, the use of these agents should be restricted to those patients who have a chronic refractory ITP [33, 41] according to the recent set of terminology criteria [1]. Whether the transient use of TPO-R agonist could be also helpful for the management of patients with severe persistent ITP still needs to be established as only a minority of these patients will eventually achieve spontaneous remission. Lastly, H. pylori eradication could be considered as rather anecdotal but, in view of the costless and safety profile of both the diagnostic methods and the eradication regimen, a systematic screening should be proposed to ITP patients at higher risk of $H$. pylori carriage, namely, living in countries with a high prevalence of $\mathrm{Hp}$ infection or above 50 years of age.

Conflicts of interest Bertrand Godeau is consultant for AMGEN France, Roche France and LFB (Laboratoire Français de Fractionnement et de Biotechnologies); he received research funds from AMGEN and Roche. Marc Michel has participated to scientific advisory boards from AMGEN and GlaksoSmithKline and has received fees as a speaker in satellite symposia for less than US\$ 10,000 .

\section{References}

1. Rodeghiero F, Stasi R, Gernsheimer T et al (2009) Standardization of terminology, definitions and outcome criteria in immune thrombocytopenic purpura of adults and children: report from an international working group. Blood 113:2386-2393

2. Neylon AJ, Saunders PW, Howard MR, Proctor SJ, Taylor PR (2003) Clinically significant newly presenting autoimmune thrombocytopenic purpura in adults: a prospective study of a population-based cohort of 245 patients. Br J Haematol 122:966974

3. Marieke Schoonen W, Kucera G, Coalson J, Li L, Rutstein M, Mowat F, Fryzek J, Kaye JA (2009) Epidemiology of immune thrombocytopenic purpura in the General Practice Research Database. Br J Haematol 145:235-244

4. Portielje JE, Westendorp RG, Kluin-Nelemans HC, Brand A (2001) A morbidity and mortality in adults with idiopathic thrombocytopenic purpura. Blood 97:2549-2554

5. Mc Millan R, Durette C (2004) Long-term outcomes in adults with chronic ITP after splenectomy failure. Blood 104:956-960 
6. Godeau B, Provan D, Bussel J (2007) Curr Opin Hematol 14:535556

7. George JN, Woolf SH, Raskob GE, Wasser JS, Aledort LM, Ballem PJ et al (1996) Idiopathic thrombocytopenic purpura: a practice guideline developed by explicit methods for the American Society of Hematology. Blood 88:3-40

8. British Committee for Standards in Haematology General Haematology Task Force (2003) Guidelines for the investigation and management of idiopathic thrombocytopenic purpura in adults, children and in pregnancy. Br J Haematol 120:574-596

9. Gasbarrini A, Franceschi F, Tartaglione R, Landolfi R, Pola P, Gasbarrini G (1998) Regression of autoimmune thrombocytopenia after eradication of Helicobacter pylori. Lancet 352:878

10. Stasi R, Provan D (2008) Helicobacter pylori and chronic ITP. Hematology Am Soc Hematol Educ Program, pp 206-211

11. Takahashi T, Yujiri T, Shinohara K, Inoue Y, Sato Y, Fujii Y, Okubo M, Zaitsu Y, Ariyoshi K, Nakamura Y, Nawata R, Oka Y, Shirai M, Tanizawa Y (2004) Molecular mimicry by Helicobacter pylori CagA protein may be involved in the pathogenesis of $H$. pylori-associated chronic idiopathic thrombocytopenic purpura. $\mathrm{Br}$ J Haematol 124(1):91-96

12. Stasi R, Sarpatwari A, Segal JB, Osborn J, Evangelista ML, Cooper N, Provan D, Newland A, Amadori S, Bussel JB (2009) Effects of eradication of Helicobacter pylori infection in patients with immune thrombocytopenic purpura: a systematic review. Blood 113:1231-1240

13. Guzman Moreno R (2009) B-cell depletion in autoimmune diseases. Advances in autoimmunity. Autoimmun Rev 8:585-590

14. Saleh MN, Gutheil J, Moore M, Bunch PW, Butler J, Kunkel L, Grillo-López AJ, LoBuglio AF (2000) A pilot study of the antiCD20 monoclonal antibody rituximab in patients with refractory immune thrombocytopenia. Semin Oncol 27(6 Suppl 12):99-103

15. Arnold DM, Dentali F, Crowther MA, Meyer RM, Cook RJ, Sigouin C, Fraser GA, Lim W, Kelton JG (2007) Systematic review: efficacy and safety of rituximab for adults with idiopathic thrombocytopenic purpura. Ann Intern Med 146:25-33

16. Godeau B, Porcher R, Fain O, Lefrère F, Fénaux P, Cheze S et al (2008) Rituximab is an alternative to splenectomy in adults with chronic immune thrombocytopenic purpura. Results of a multicenter prospective phase 2 study. Blood 112:999-1004

17. Zaja F, Baccarani M, Mazza P, Bocchia M, Gugliotta L, Zaccaria A, Vianelli N, Defina M, Tieghi A, Amadori S, Campagna S, Ferrara F, Angelucci E, Usala E, Cantoni S, Visani G, Fornaro A, Rizzi R, De Stefano V, Casulli F, Battista ML, Isola M, Soldano F, Gamba E, Fanin R (2010) Dexamethasone plus rituximab yields higher sustained response rates than dexamethasone monotherapy in adults with primary immune thrombocytopenia. Blood 115:2755-2762

18. Zaja F, Battista ML, Pirrotta MT, Palmieri S, Montagna M, Vianelli $\mathrm{N}$ et al (2008) Lower dose rituximab is active in adult patients with idiopathic thrombocytopenic purpura. Haematologica 93:930-933

19. Provan D, Butler T, Evangelista ML, Amadori S, Newland AC, Stasi R (2007) Activity and safety profile of low-dose rituximab for the treatment of autoimmune cytopenias in adults. Haematologica 92:1695-1698

20. Wegener A, Liebman HA, Saleh MN, Abassi R, Bussel J, Cosgriff TM, Horne H, Wolffgram T, Goldenberg DM (2009) Multicenter study of low-dose anti-CD20 veltuzumab for treatment of relapsed immune thrombocytopenia. Heamatologica 94(suppl2):3 abs.0227

21. Stasi R, Del Poeta G, Stipa E, Evangelista ML, Trawinska MM, Cooper N et al (2007) Response to B-cell depleting therapy with rituximab reverts the abnormalities of T-cell subsets in patients with idiopathic thrombocytopenic purpura. Blood 110:2924-2930

22. Stasi R, Cooper N, Del Poeta G, Stipa E, Laura Evangelista M, Abruzzese E, Amadori S (2008) Analysis of regulatory T-cell changes in patients with idiopathic thrombocytopenic purpura receiving $\mathrm{B}$ cell-depleting therapy with rituximab. Blood 112: $1147-1150$

23. Zaja F, Vianelli N, Battista M, Sperotto A, Patriarca F, Tomadini V, Filì C, Tani M, Baccarani M, Fanin R (2006) Earlier administration of rituximab allows higher rate of long-lasting response in adult patients with autoimmune thrombocytopenia. Exp Hematol 34:571-572

24. Shenoy S, Mohanakumar T, Chatila T, Tersak J, Duffy B, Wang R, Thilenius AR, Russell JH (2001) Defective apoptosis in lymphocytes and the role of IL-2 in autoimmune hematologic cytopenias. Clin Immunol 99:266-275

25. Olsson B, Andersson PO, Jacobsson S, Carlsson L, Wadenvik H (2005) Disturbed apoptosis of T-cells in patients with active idiopathic thrombocytopenic purpura. Thromb Haemost 93:139144

26. Medeot M, Zaja F, Vianelli N, Battista M, Baccarani M, Patriarca F, Soldano F, Isola M, De Luca S, Fanin R (2008) Rituximab therapy in adult patients with relapsed or refractory immune thrombocytopenic purpura: long-term follow-up results. Eur J Haematol 81:165-169

27. Patel V, Mihatov N, Cooper N, Stasi R, Cunningham-Rundles S, Bussel JB (2006) Long term follow-up of patients with immune thrombocytopenic purpura whose initial response to rituximab lasted a minimum of 1 year. 48th Annual Meeting and Exposition of the American Society of Hematology, December 9-12, Orlando, FL, abstract 479

28. Cooper N, Stasi R, Cunningham-Rundles S, Feuerstein MA, Leonard JP, Amadori S, Bussel JB (2004) The efficacy and safety of B-cell depletion with anti-CD20 monoclonal antibody in adults with chronic immune thrombocytopenic purpura. Br J Haematol $125: 232-239$

29. Edwards JC, Cambridge G, Leandro MJ (2006) B cell depletion therapy in rheumatic disease. Best Pract Res Clin Rheumatol 20:915-928

30. Tsutsumi Y, Kanamori H, Mori A, Tanaka J, Asaka M, Imamura M, Masauzi N (2005) Reactivation of hepatitis B virus with rituximab. Expert Opin Drug Saf 4:599-608

31. Calabrese LH, Molloy ES, Huang DR, Ransohoff RM (2007) Progressive multifocal leukoencephalopathy in rheumatic diseases. Arthritis Rheum 56:2116-2128

32. Carson KR, Evens AM, Richey EA, Habermann TM, Focosi D, Seymour JF et al (2008) Progressive multifocal leukoencephalopathy after rituximab therapy in HIV-negative patients: a report of 57 cases from the Research on Adverse Drug Events and Reports project. Blood 113:4834-4840

33. Gernsheimer TB (2008) The pathophysiology of ITP revisited: ineffective thrombopoiesis and the emerging role of thrombopoietin receptor agonists in the management of chronic immune thrombocytopenic purpura. Hematology Am Soc Hematol Educ Program 219-226

34. Kuter DJ, Bussel JB, Lyons RM et al (2008) Efficacy of romiplostim in patients with chronic immune thrombocytopenic purpura: a double-blind randomised controlled trial. Lancet 371 (9610):395-403

35. Bussel JB, Kuter DJ, Pullarkat V, Lyons RM, Guo M, Nichol JL (2009) Safety and efficacy of long-term treatment with romiplostim in thrombocytopenic patients with chronic ITP. Blood 113:2161-2171, Erratum in: Blood. 2009 May 7;113(19):4822

36. Bussel JB, Cheng G, Saleh MN et al (2007) Eltrombopag for the treatment of chronic idiopathic thrombocytopenic purpura. N Engl J Med 357:2237-2247

37. Bussel JB, Provan D, Shamsi T, Cheng G, Psaila B, Kovaleva L et al (2008) Effect of eltrombopag on platelet counts and bleeding during treatment of chronic idiopathic thrombocytopenic purpura: a randomised, double-blind, placebo-controlled trial. Lancet 373 (9664):641-648 
38. Podolanczuk A, Lazarus AH, Crow AR, Grossbard E, Bussel JB (2009) Of mice and men: an open-label pilot study for treatment of immune thrombocytopenic purpura by an inhibitor of Syk. Blood 113:3154-3160

39. Patel VL, Schwartz J, Bussel JB (2008) The effect of anti-CD40 ligand in immune thrombocytopenic purpura. $\mathrm{Br} \mathrm{J}$ Haematol $141: 545-548$
40. Kuwana M, Nomura S, Fujimura K, Nagasawa T, Muto Y, Kurata Y, Tanaka S, Ikeda Y (2004) Effect of a single injection of humanized anti-CD154 monoclonal antibody on the plateletspecific autoimmune response in patients with immune thrombocytopenic purpura. Blood 103:1229-1236

41. George JN (2009) Definition, diagnosis and treatment of immune thrombocytopenic purpura. Haematologica 94:759-762 